\title{
Transport of large particles through the transition to turbulence of a swirling flow
}

\author{
Nathanaël Machicoane $\mathbb{1}^{1, *}$ and Romain Volk ${ }^{2}$ \\ ${ }^{1}$ LEGI, CNRS, Université Grenoble Alpes, Grenoble INP, 38000 Grenoble, France \\ ${ }^{2}$ Laboratoire de Physique, CNRS, École Normale Supérieure de Lyon, Université de Lyon, \\ 46 Allée d'Italie, 69364 Lyon Cedex 07, France
}

(Received 19 December 2020; accepted 2 April 2021; published 16 April 2021)

\begin{abstract}
We investigate the behavior of large particles in a transitional swirling flow in a closed vessel, focusing on both their transport and flow sampling. We conduct a Lagrangian study of slightly buoyant particles, considering three particle diameters, keeping the fluid's density and velocity magnitude constant and varying the Reynolds number by changes of the fluid's viscosity. The literature lacks study of material particles in such flows, where chaos and bursts of turbulence can exist, despite applications in industrial and natural situations. One striking result is that particles beyond a certain size, between 10 and $18 \mathrm{~mm}$, are subject to a strong trapping in the vicinity of the islands of the laminar flow, while large particles below this threshold sample the flow homogeneously, independently of the Reynolds number. The exploration of the flow by the large particles is widely different from the preferential sampling in fully developed turbulent conditions, in terms of characteristic times associated with motions escaping the regions and dimensions of the sampled regions. While the particle mean velocity is found to be independent of both the fluid's viscosity and particle size, the fluctuation magnitude strongly increases with decreasing flow viscosity and is marginally affected by size. We also characterize the intensity of the trapping through probability density functions of the particle positions, a measure of the dimensions of the sampled regions, and particle position autocorrelation functions as an attempt to quantify residence times. A qualitative origin for the trapping existence criterion based on a shear-induced lift force is proposed, as an argument for its sole dependence on particle size.
\end{abstract}

DOI: 10.1103/PhysRevFluids.6.044303

\section{INTRODUCTION}

The mechanisms governing transport of particles and mixing play a tremendous role in many natural and industrial situations, such as pollutant dispersion, accumulation of plastics in the ocean, food processing, and propulsion, to name a few. Many of these applications deviate strongly from the canonical study cases that are well understood. While mixing has been studied in detail in a wide scope of conditions, including laminar, chaotic, and turbulent, in various geometries [1], a much narrower scope has been covered concerning particle dynamics. The latter has indeed received a great deal of attention in the past couple of decades, but with a strong focus on small particles in homogeneous isotropic turbulence [2]. When the particles are smaller than the flow length scales (being smaller or of the order of the Kolmogorov length for turbulent conditions) but the particle dynamics deviates from the one of the fluid's particle due to a difference in density, they are referred to as inertial particles. Particles whose response time differs due to a larger size or a

\footnotetext{
*nathanael.machicoane@univ-grenoble-alpes.fr
} 
combination of large size and different density are called material particles [3]. In many applications, the objects being transported often lie in this class of particles and the carrier flows often present a large-scale structure, either due to a close geometry or the proximity to boundaries. Recent efforts have been carried out, characterizing the dynamics of material particles in turbulent flows [4-10], but often without a large-scale flow structure or using Reynolds decomposition approaches in its presence. For a strongly inhomogeneous turbulent flow, the large-scale structure has been shown to induce a preferential sampling of the flow by the large particles when their size exceeds a critical diameter, with back-and-forth motions between the explored regions for long times $[11,12]$. When other forces are involved in the particle dynamics, such as gravity, similar preferential sampling around equilibrium points can take place even in more canonical configurations such as a free jet [13]. This present study addresses the questions of the material particles dynamics in a closed flow for transitional regimes, aiming at filling the gap between the studies for fully developed turbulent conditions and the understanding in laminar cases [14-16]. Besides advancing our understanding of large object transport for the cited applications, we hope this contribution can also help validate recent numerical advances of transport of (resolved) material particles ([17] for instance).

The configuration chosen to study tranport of large particles in transitional inhomogeneous flows is the one produced by two counterrotating disks in a closed container, referred to as von Kármán swirling flow. Abundant literature can be found concerning the flow itself, under laminar, transitional, and turbulent conditions (detailed below), as well as concerning particle transport in turbulence. The supercritical transition of the von Kármán swirling flow from a laminar to a fully developed turbulent state as the Reynolds number increases was studied in detail for a circular cross section $[18,19]$. For small Reynolds numbers $(\operatorname{Re}<350$, based on the disk radius and tip speed and for disks with blades), the flow is laminar and two counterrotating toroidal cells as well as two poloidal recirculations are observed. When the Reynolds number is between 350 and 2500, the flow transits to turbulence [19]. Temporal velocity fluctuation appears around the mean structure described above and the velocity frequency spectra, which mostly show a peak around the forcing frequency in the laminar state, have a higher plateau value with a decay range at high frequencies; many other peaks appear as the Reynolds number increases. For $\operatorname{Re}>2500$, the spectra show a scale continuum (also confirmed by direct numerical simulations resolving the propellers [20]), as the flow is turbulent. The magnitude of the velocity fluctuations, starting at 0 for $\operatorname{Re}<350$, increases rapidly in a supercritical turbulent transition (as a function of $\sqrt{\mathrm{Re}}$ ) and for $\operatorname{Re}>3100$ shows a plateau along Re when normalized by the disk tip speed.

For laminar conditions, the center of the flow is a stagnation point and a fluid particle cannot move across the median plane that separates both toroidal cells [see Fig. 1(a)]. The particles' attractors seem to closely match the locations of the centers of the poloidal recirculations, which are neutral poloidal lines. In the vicinity of the neutral lines are flow islands, regions where a scalar field would not be advected out of and would only spread out through molecular diffusion [21]. The three-dimensional (3D) structure of the laminar flow can hence be decomposed into chaotic regions, from which fluid particles and inertial particles are ejected, and islands, where both particle types have been shown to be trapped over extremely long times in similar geometries [22,23]. Escauriaza and Sotiropoulos [23] studied in particular the vicinity of flow islands and decomposed them for the fluid's particles as toroidal invariant regions, and narrow bands of periodic orbits nested and folded near the boundary of the torus. These regions have been evidenced to exist for inertial particles three decades ago [24], and another common example where they have been reported is the Taylor-Couette flow [25]. In the case studied by Escauriaza and Sotiropoulos [23], the flow is three dimensional but fully laminar and no fluctuations are present (they considered smooth disks, increasing the threshold Reynolds number for unsteadiness to $\mathrm{Re}=850$ ). Inertial particles can only escape the no-mixing regions due to a non-negligible settling velocity. They indeed consider a circular section cylindrical vessel with two counterrotating disks whose axis is parallel to gravity and show that the mixing efficiency depends strongly on the particle settling velocity. For a given laminar flow where the fluid's particles experience chaotic Lagrangian behavior, they showed that the stirring rates depend strongly on particle parameters for the model inertial particles considered 
(a)

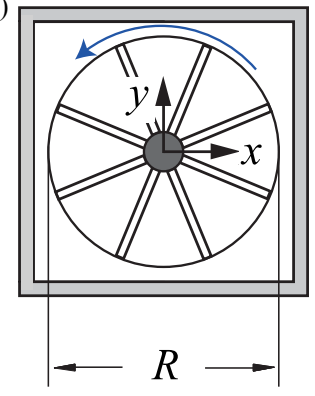

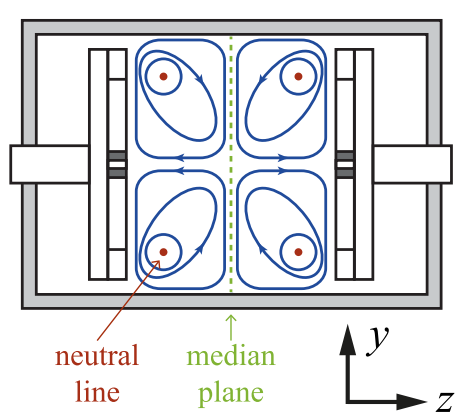

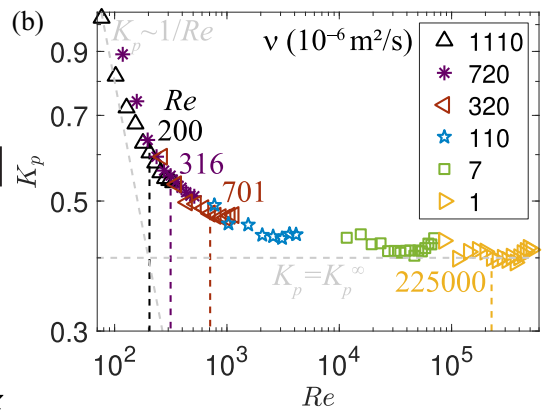

FIG. 1. (a) Slices of the von Kármán square vessel showing a disk and the space between the disks with a schematic of the mean flow (only the poloidal component is shown in this plane). The median plane $(x, y, z=0)$, separating each toroidal counterrotating cell, and the poloidal neutral lines are represented. The latter correspond to the center of the poloidal recirculations and are located around $|z|=0.7 R$ and $r=\sqrt{x^{2}+y^{2}}=0.75 R$. (b) Nondimensional torque of the motors $K_{p}=\varepsilon V / R^{5}(2 \pi \Omega)^{3}$ as a function of the large-scale Reynolds number $\mathrm{Re}=2 \pi R^{2} \Omega / v$ for various fluid kinematic viscosities (symbols and colors) and increasing values of the disk frequency. The four vertical lines indicate the Reynolds number values for $\Omega=4 \mathrm{~Hz}$ for the conditions of Table I (with two extra conditions to capture the trends, 4 and $\star$, and water-glycerol mixtures of volume ratios $33: 77$ and 50:50 at temperatures $0^{\circ} \mathrm{C}$ and $20^{\circ} \mathrm{C}$ ). The dashed gray lines illustrate the asymptotic trends for low and high Reynolds numbers $K_{p} \sim 1 / \operatorname{Re}$ and $K_{p}=K_{p}^{\infty}$, respectively.

in this numerical study. Whether this statement would hold for material particles or for laminar conditions and how trapping in the vicinity of flow islands would change as the flow becomes transitional are open questions.

This article aims at studying the dynamics of large particles in the von Kármán swirling flow in the range $200<\operatorname{Re}<700$, exploring laminar and transitional conditions. Section II describes the experimental setup, the fluids and particles used, as well as the measurement methods and analysis tools. A characterization of the particle dynamics and of the trapping in the vicinity of the flow islands is then made in Secs. III and IV, respectively. Arguments for the difference in the dynamics below and above the critical size for trapping existence, as well as for the possible origin of the trapping, are then discussed in Sec. V). A summary follows in Sec. VI.

\section{EXPERIMENTAL SETUP AND METHODS}

\section{A. Transition to turbulence in a swirling flow}

The von Kármán swirling flow has been used extensively in the past for the study of particle dynamics in fully developed turbulence [2]. A fluid contained in a closed vessel is stirred by two counterrotating disks, producing a strong mean flow with two toroidal cells of opposite azimuthal velocity and two poloidal recirculations due to the disks' pumping, both being separated by the median plane. A square section vessel and disks with straight blades are used in the current study [see Fig. 1(a) and [11]]. For laminar conditions, only the mean flow exists and no velocity fluctuations are present. For fully turbulent conditions, the velocity fluctuations can reach up to one-third of the disk tip speed $U=2 \pi R \Omega$, where $R$ and $\Omega$ are the disk radius and rotating frequency, respectively, and both the mean flow and the fluctuations are highly anisotropic $[26,27]$. Following the studies in circular section vessels [18,19], we use fluids of different kinematic viscosity $v$ values, vary the disk rotating frequency to span a wide range of large-scale Reynolds numbers $\operatorname{Re}=U R / \nu=2 \pi R^{2} \Omega / \nu$, and use the total motors' power, divided by the fluid's mass, as a proxy for the energy dissipation rate $\varepsilon$. Variation of the fluid viscosity is reached by use of pure glycerol, water-glycerol mixtures, and pure water, with possible variation of the temperature (using a thermal 
TABLE I. Flow parameters for a disk rotation frequency $\Omega=4 \mathrm{~Hz}$, with different values of the kinematic viscosity $v$ obtained with pure glycerol $\left(99 \%\right.$ purity) at $20^{\circ} \mathrm{C}, 25^{\circ} \mathrm{C}$, and $35^{\circ} \mathrm{C}$, respectively, and water at $20^{\circ} \mathrm{C}$ (for lines 1-4, respectively). The large-scale Reynolds number is defined as $\operatorname{Re}=2 \pi R^{2} \Omega / \nu$, with $R=9.5 \mathrm{~cm}$ the disk radius; $\varepsilon$ is the energy dissipation rate (per unit mass) estimated based on the power consumption of the motors that rotate the disks; $\beta=3 \rho_{f} /\left(\rho_{f}+2 \rho_{p}\right)$ is the particle density ratio, with indices $f$ and $p$ for the particle and the fluid, with $\rho_{p}=0.9 \times 10^{3}$ and $1.14 \times 10^{3} \mathrm{~kg} / \mathrm{m}^{3}$ for water and glycerol, respectively.

\begin{tabular}{lccr}
\hline \hline$v$ & $\operatorname{Re}$ & $\begin{array}{c}\varepsilon \\
(\mathrm{W} / \mathrm{kg})\end{array}$ & $\beta$ \\
$10^{-6}\left(\mathrm{~m}^{2} / \mathrm{s}\right)$ & & 6.4 & 1.07 \\
1110 & 200 & 5.85 & 1.07 \\
720 & 316 & 5.04 & 1.06 \\
320 & 701 & 4.03 & 1.07 \\
1 & 225000 & & \\
\hline \hline
\end{tabular}

bath with a water circulation in the shafts of the vessel; see [28] for more details). The adimensional torque is then computed as $K_{p}=\varepsilon V / R^{5}(2 \pi \Omega)^{3}$, where $V$ is the vessel's volume, and is reported for six values of viscosity in Fig. 1(b).

Both asymptotic regimes $K_{p} \sim 1 / \operatorname{Re}$ and $K_{p}=K_{p}^{\infty}$, for low and high values of the Reynolds number, respectively, are represented. For $\operatorname{Re}<100$, the flow is fully laminar and $\varepsilon \sim \Omega^{2}$, while for $\operatorname{Re}>10^{5}$ fully developed turbulence leads to $\varepsilon \sim \Omega^{3}$ [Fig. 1(b)]. We chose to study the dynamics of large particles in this swirling flow in the transition to the turbulence regime, where $K_{p}$ does not follow any of the asymptotic trends. As the particle used deviates slightly from neutrally buoyant $\left(\rho_{p}=1.14 \times 10^{3} \mathrm{~kg} / \mathrm{m}^{3}\right.$, while $\left.\rho_{f} \simeq 1.26 \times 10^{3} \mathrm{~kg} / \mathrm{m}^{3}\right)$, a fixed rotating frequency of $\Omega=4 \mathrm{~Hz}$ is chosen, so the particles' typical velocity (about $0.25 U=0.6 \mathrm{~m} / \mathrm{s}$ ) is much greater than their settling velocity (at most $0.4 \mathrm{~mm} / \mathrm{s}$ ). The variation in Reynolds number is then achieved by using pure glycerol (99\% purity), at three different temperatures, changing $v$ by a factor of about 3.5, while the fluid's density stays roughly constant (see Table I). To provide a comparison with the dynamics of such particles in fully developed turbulence, we use slightly light particles $\left(\rho_{p}=0.9 \times 10^{3} \mathrm{~kg} / \mathrm{m}^{3}\right)$ in water with $\operatorname{Re}=225000$ (see [7] for more details). The density ratio $\beta=3 \rho_{f} /\left(\rho_{f}+2 \rho_{p}\right)$, which is commonly used in turbulence as it appears in added mass, is in all cases considered $\beta=$ $1.07 \pm 0.01$.

The large particles considered are spherical, with diameters of 10, 18, and $24 \mathrm{~mm}$. Only one particle of each size is in the vessel at the same time, so no particle-particle interactions are present. In fully developed turbulence, such large particles, whether neutrally buoyant or slightly light or heavy, experience a preferential sampling of the flow [12]. They are more likely to be found in low-fluctuation, high-mean-velocity regions, in particular in the counterrotating toroidal cells, on the side of the vessel, discriminating only the axial $z$ exploration and not the radial exploration of the vessel. While lighter particles experience slightly enhanced preferential sampling (longer residence times on a given side of the vessel), the criterion for exhibiting such behavior has been shown to be only particle size, with a critical size lying in between 10 and $18 \mathrm{~mm}$ for the given geometry, in relation to the mean flow structure characteristic scales.

\section{B. Particle tracking}

Using the same optical setup as in [11], with two cameras at a $90^{\circ}$ angle [one facing the $(x, z)$ plane and the other the $(y, z)$ plane], with a field of view covering the whole vessel, we can apply classic stereomatching and reconstruct the individual particle trajectories $\mathbf{X}(t)$. We note that both transverse components $x$ and $y$ play similar roles in the current geometry and only $x$ will be reported in the statistical analysis of the following sections. For each condition, a collection of 170 movies is recorded, with a high frame rate $(500-1500 \mathrm{~Hz}$ as Re increases from 200 to 701). Due to the limitation of the cameras' on-board memory, their duration ranges from 30 to $10 \mathrm{~s}$ (as the frame 
rate increases). This results in a track duration of several hundred to more than 1000 integral time $T_{\text {int }}=1 / \Omega$, corresponding to over $2 \times 10^{6}$ sampled points, per particle and per flow Reynolds number.

Figure 2 plots a sample 3D trajectory for a particle of $18 \mathrm{~mm}$ for the three Reynolds numbers considered and a fixed duration $T \Omega=40$. The median plane is represented to help differentiate each side of the vessel (the disks being at $z= \pm 1.05 R$ ). For $\operatorname{Re}=200$, the particle stays on one side of the vessel for the whole trajectory (while this is not represented in the figure, the particles remains on this side of the vessel for at least the whole duration of the trajectory, $T \Omega=120$ ). The particle stays in the vicinity of the poloidal neutral line (defined as $|z|=0.7 R$ and $r=\sqrt{x^{2}+y^{2}}=0.75 R$ ), its trajectory twisting along the way around it, so that $Z=0.65 \pm 0.1 R$ and $r=0.8 \pm 0.1 R$ (estimated from the mean and standard deviation of the particle positions). We note that inertial particles in a similar flow exhibit very similar attractors [23] and such chaotic behavior is in fact common in flows in cavities [1]. For this sample trajectory, it takes about 3.5 disk rotations $(0.9 \mathrm{~s})$ for the particle to rotate $360^{\circ}$ around the $z$ axis (and come close to its original position). During this revolution, its axial $Z$ position oscillates twice around its mean position $|Z|=0.7 R$. This indicates quasiperiodicity in the particle dynamics, with the main frequencies of axial motions that are about twice the ones associated with transverse motions. For $\mathrm{Re}=316$, while a few closed orbits, reminiscent of the one seen at $\operatorname{Re}=200$, can be observed, the particle explores a wider range of positions within a given side of the vessel and can be seen escaping it once to rapidly move to the opposite side. At $\operatorname{Re}=701$, no closed orbit is noted, meaning that the particle does not return to a given original position in a correlated fashion, but only after many dozens of turnover times $1 / \Omega$. The exploration of each side of the vessel is much enhanced. Note that the exploration of the median plane vicinity is never expected here for such large particles, as it does not happen for fully developed conditions, where large particles already preferentially sample the side of the vessel [11]. Even in this context, the median plane is only briefly passed through, as it is an unstable fixed point [29], and particles can remain on a given side of the vessel for long times [12].

To quantify the exploration of a given side of the vessel as a function of the Reynolds number, Fig. 2(d) presents the standard deviation of the axial position absolute value $|Z|^{\prime}$. Computation of only the axial position standard deviation $Z^{\prime}$ would not represent the width of domain explored on a given side, as excursions to the opposite side would bias the quantity and make it erroneously large. The 10-mm particle samples the vessel in a roughly homogeneous fashion even with the very low level of the fluid's velocity fluctuations. Its flow sampling in the laminar flow is actually very similar to what is observed in the fully developed turbulent flow [further discussed below; see Fig. 6(d)], indicating that it is independent of the Reynolds number. This means that, beyond being independent of the particle density ratio (in a range $0.9<\beta<1.1$ ), the criterion for the existence of preferential sampling is also independent of the Reynolds number. For particles above the critical size, which lies in between 10 and $18 \mathrm{~mm}$ for the considered geometry, the intensity of the trapping depends strongly on the Reynolds number. The width of the sampled regions within a side of the vessel is reduced by a factor of about 2 for $\operatorname{Re}=200$ compared to $\operatorname{Re}=701$ for both 18 and $24 \mathrm{~mm},|Z|^{\prime}(\operatorname{Re})$ showing a similar behavior for both sizes (the value is only slightly higher for $18 \mathrm{~mm}$ at $\mathrm{Re}=701$ and identical for lower Reynolds numbers).

Figure 2(e) represents a sample trajectory for a 10-mm particle at the lowest Reynolds number considered. The sampling of the vessel appears much more homogeneous than the one done by the 18-mm particle in the transitional flow $[\mathrm{Re}=701$, Fig. 2(c)]. This is a qualitative illustration of the drastic differences in the dynamics between particles below and above the critical size. Figure 2(f) besides displays a schematic representation of a cut of the mean flow in the laminar regime, in half of the vessel cross section, obtained through an Eulerian conditioning of the Lagrangian tracks followed by an azimuthal integration. In fully developed turbulence, the mean local particle velocity has been shown to be independent of particle size [11] and we assume this holds in laminar conditions, which allows us to use it as a proxy for the mean flow. 

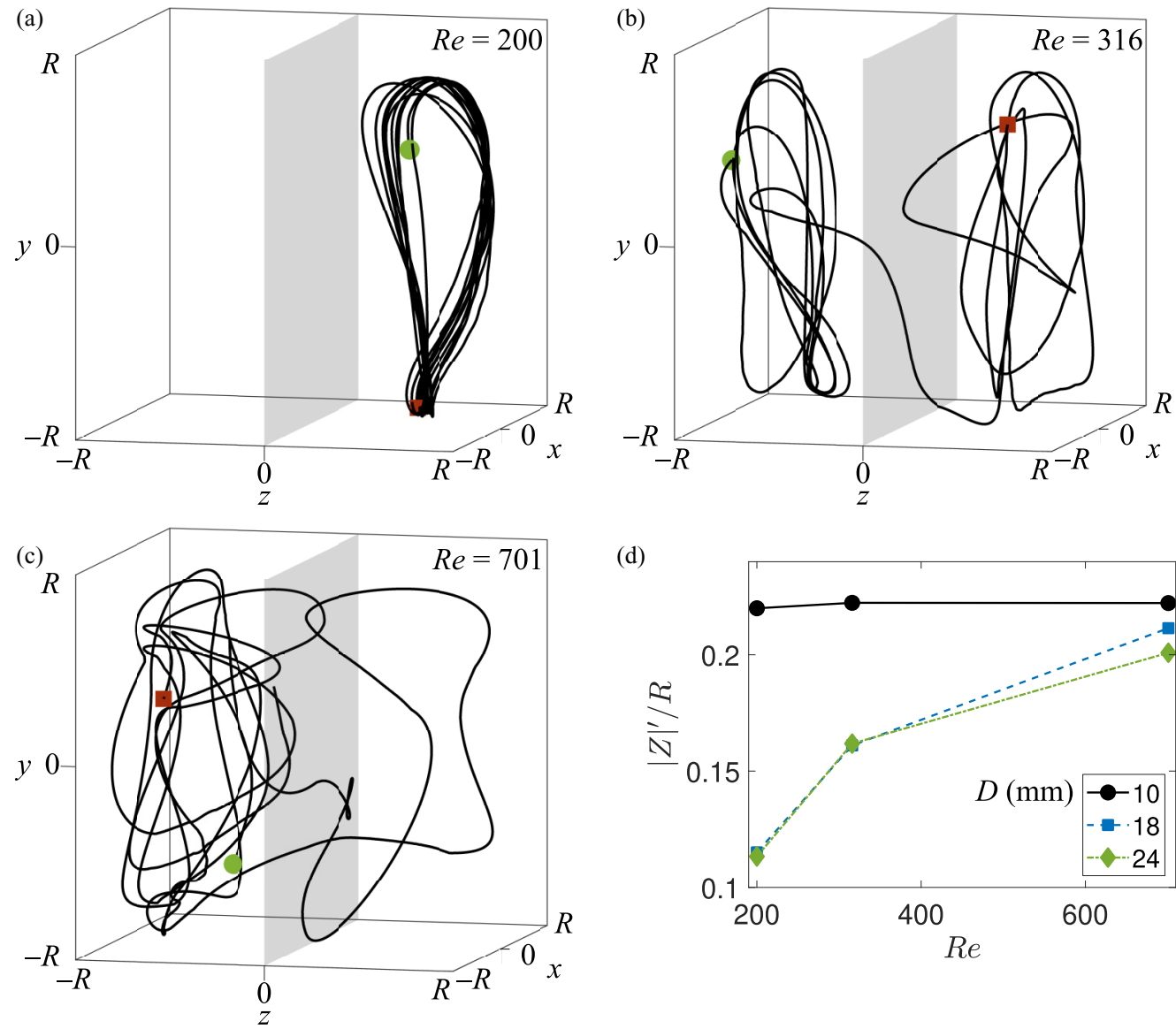

(d)
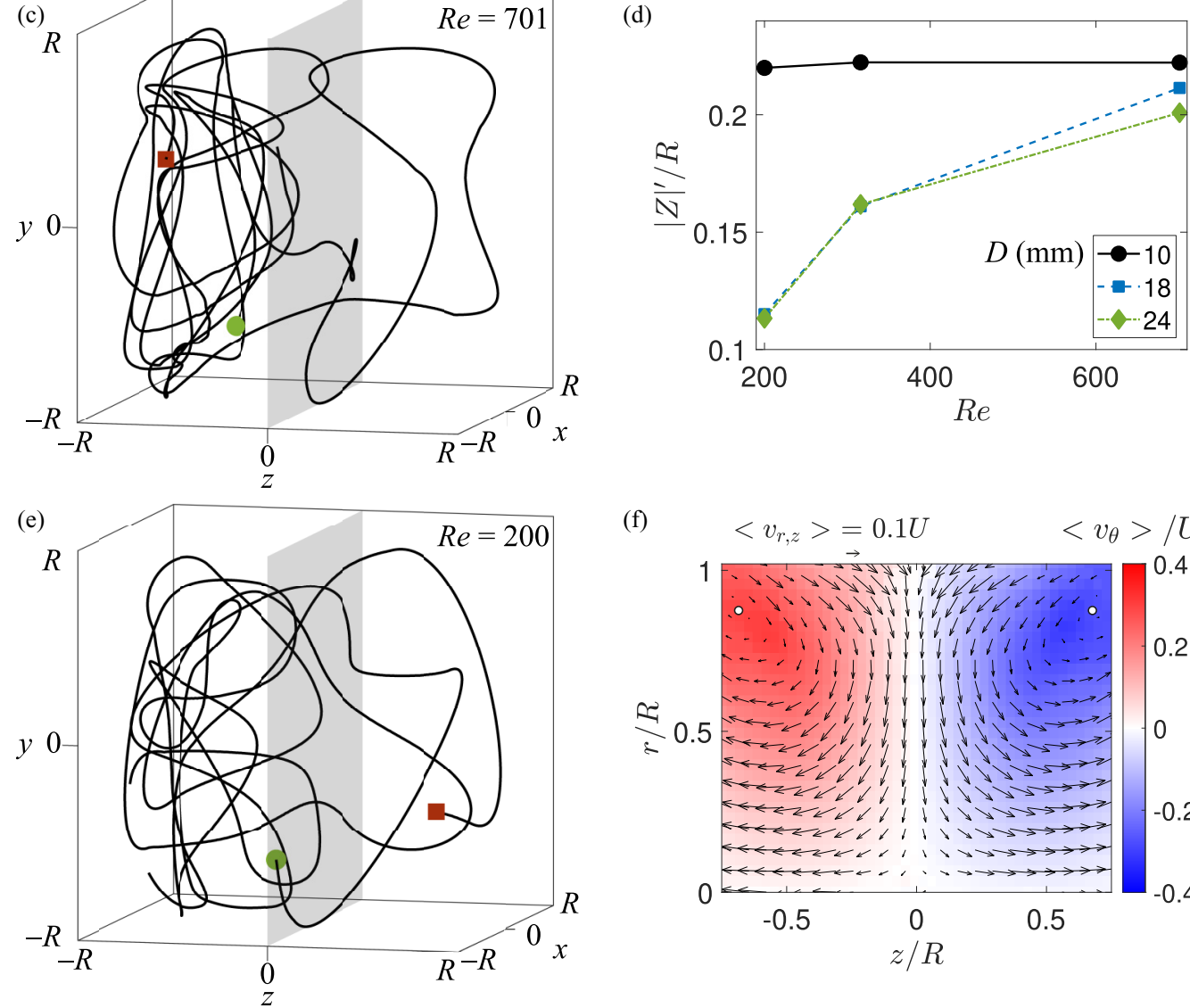

(f)

$$
<v_{r, z}>=0.1 U \quad<v_{\theta}>/ U
$$

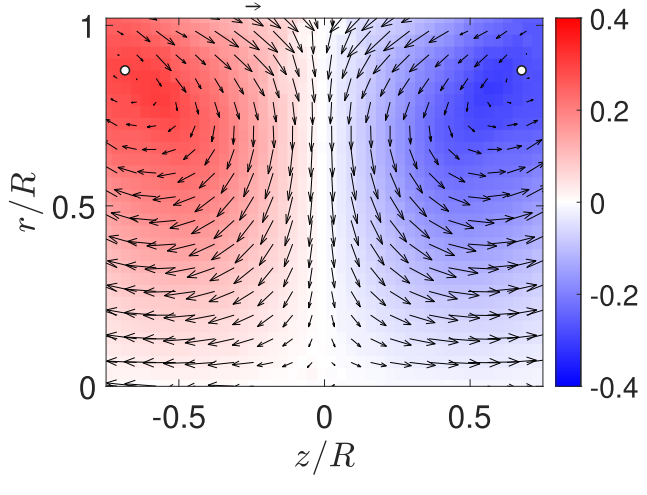

FIG. 2. (a)-(c) Sample trajectory for a particle of $18 \mathrm{~mm}$, with a fixed duration $T \Omega=40$ and for the three Reynolds number values considered. The start and end points of the trajectories are indicated by and $\bullet$, respectively. The median plane is indicated by a semitransparent gray plane. (d) Standard deviation of the absolute value of the collection of $Z$ positions for all trajectories as a function of Re, for each particle diameter. (e) Sample trajectory for a particle of $10 \mathrm{~mm}$, with a fixed duration $T \Omega=40$ for $\operatorname{Re}=200$. (f) Mean velocity field of a $10-\mathrm{mm}$ particle at $\mathrm{Re}=200$ in the half cross section of the vessel, obtained by an Eulerian conditioning of the Lagrangian tracks followed by an azimuthal integration (see [11] for more details). The white circles indicate the locations of the poloidal neutral lines. 

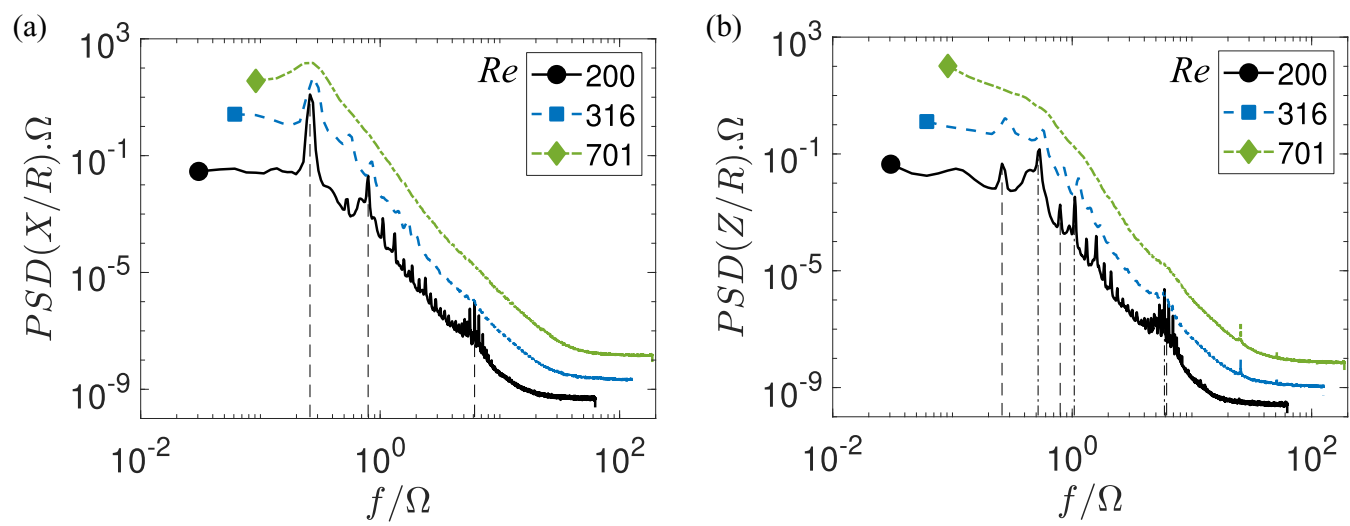

FIG. 3. Power spectral densities of the (a) transverse $X$ and (b) axial $Z$ particle positions, for a particle of $18 \mathrm{~mm}$, and different Reynolds numbers, subsequently multiplied by a factor 10 for visibility. The peaks identified in (a) by dashed lines are at $f=[0.259,0.794,6.15] \Omega$. The peaks identified in (b) by dot-dashed lines are at $f=[0.519,1.05,5.89] \Omega$ and the dashed lines from (a) are reported as well.

\section{PARTICLE DYNAMICS}

From the collection of particle trajectories $\mathbf{X}(t)$, we compute average power spectral densities (PSDs) to study the particle temporal dynamics. Figures 3(a) and 3(b) show the particle $(D=18 \mathrm{~mm})$ transverse and axial position PSDs for the three Reynolds numbers considered (each spectrum being shifted by one decade for clarity). The transverse component PSD presents independently of the Reynolds number a plateau at low frequencies $f<0.1 \Omega$, representing total decorrelation of the particle transverse position at long times, while the range $0.5<f / \Omega<5$ has a power-law decay, with an exponent approximately $-5 \pm 0.5$. The high-frequency range then shows the white nature of the measurement noise of particle position. The axial component PSD has a similar general behavior, except at low frequency for $\mathrm{Re}=701$, where the PSD increases at decreasing values of $f$, indicating a long-time dynamics. This long-time dynamics, studied in detail for the case of a fully developed turbulent flow [12], is associated with the back-and-forth motion of the particle between each side of the vessel, where it can stay for long times compared to the turnover time $1 / \Omega$. For the 18 -mm particle considered here, a power-law regime, with an exponent close to -1 , can be observed. The existence of the long-time dynamics is only indicated by the first couple of points of the PSD for lower Reynolds numbers due to experimental limitations: The camera limited on-board memory was not sufficient to capture most of the back-and-forth motions in these cases, where they happen on timescales of the order of several tens of seconds. At lower Reynolds numbers, as the trapping becomes stronger, such back-and-forth motions are sparser and happen on longer and longer times, so no signature of this suspected long-time dynamics can be seen on the PSD.

Both transverse and axial components PSD present peaks, pronounced for $\operatorname{Re}=200$ and less and less so as the Reynolds number increases (to be barely a bump for $R e=701$ ). The peaks at low Reynolds numbers are signatures of the quasiperiodicity discussed above. On the one hand, the peaks observed for $\operatorname{Re}=200$ on the $x$ component are at $f=[0.259,0.794,6.15] \Omega$, respectively. The first peak, which is the dominant one, is compatible with the observation that the particle takes about 3.5 disk rotations to complete a full rotation around the $z$ axis for the sample trajectory of Fig. 2(a). This frequency can also be estimated from the observation that the particle average location and azimuthal velocity are approximately $r=0.8 R$ and $\left\langle v_{\theta}\right\rangle=0.25 U$, respectively [see Fig. 4(a)], balancing $2 \pi r f$ with $0.25 U$. The secondary peaks are expected to be harmonics of the dominant frequency, being located at 3 times and 24 times $f=0.259 \Omega$, respectively. These three peaks can also be found on the axial component PSD, with attenuated amplitudes. The dominant 

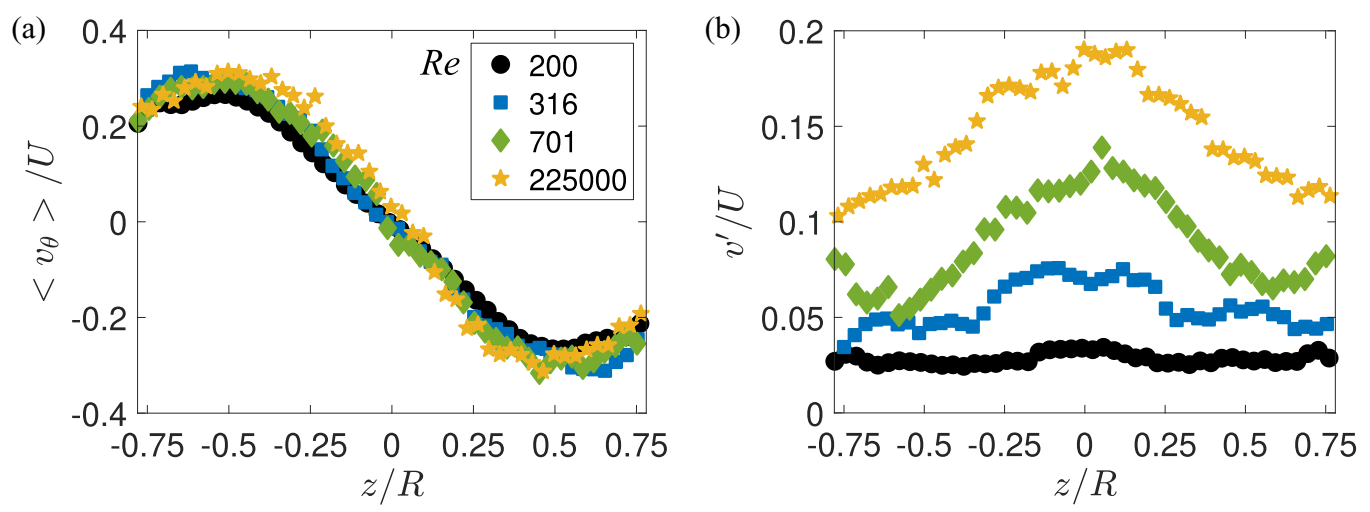

FIG. 4. (a) Local average and (b) standard deviation of the velocity extracted from an Eulerian conditioning of the Lagrangian tracks for a particle of $10 \mathrm{~mm}$, for $r=0.8 R$. The quantities are integrated azimuthally to increase statistical convergence, changing the frame of reference from Cartesian $(x, y, z)$ to cylindrical $(r, \theta, z)$ for this figure. (a) Azimuthal component of the mean particle velocity and (b) particle velocity fluctuation magnitude $v^{\prime}=\sqrt{\left(v_{r}^{\prime 2}+v_{\theta}^{\prime 2}+v_{z}^{\prime 2}\right) / 3}$.

peak is $f=0.519 \Omega$, which corresponds almost exactly to twice $f=0.259 \Omega$ and is in agreement with our observation that while the particle orbits once around the $z$ axis, $Z(t)$ experiences two periods. The two other peaks that can be noted on this spectrum are at $f=[1.05,5.89] \Omega$, twice and 11 times the dominant frequency, confirming the observed quasiperiodicity. On the other hand, the high-Reynolds-number case presents much smoother spectra, which can be interpreted as a scale continuum. This is a signature of the transition to turbulence of the carrier flow, as shown in $[18,19]$.

From the particle trajectories with positions $\mathbf{X}(t)$ at a high sample rate, we compute the particle velocity along the tracks v. Following [11], we then interpolate the Lagrangian statistics on an Eulerian grid. This simply corresponds to gathering the particle velocities passing through a given bin, which can yield, for instance, the local mean or standard deviation of the particle velocity (see Fig. 4 for a particle diameter of $10 \mathrm{~mm}$ ). The maps are integrated azimuthally to increase the statistics, yielding quantities in a cylindrical coordinate system $(z, r, \theta)$ (see [11] for more details). From these maps, Fig. 4 shows a cut of the azimuthal component of the mean particle velocity $\left\langle v_{\theta}\right\rangle(r=0.8 R, z)$. The particle mean velocity can be considered to be independent of the Reynolds number (or rather the fluid's viscosity, for a constant disk rotation frequency $\Omega$ ), as barely any change is noticed for the three Reynolds numbers considered. The condition of fully developed turbulence is added for comparison and the low-Reynolds-number profiles are very similar, only presenting a slightly less steep gradient across the median plane while conserving a similar peak velocity of about $0.28 \mathrm{U}$. Similar observations are made for all particle sizes considered and velocity components, as the mean velocity is independent of particle size (not shown here) (similarly found in fully developed turbulence [11]).

Figure 4(b) displays a cut of the particle $(D=10 \mathrm{~mm})$ velocity fluctuation magnitude $v^{\prime}=$ $\sqrt{\left(v_{r}^{\prime 2}+v_{\theta}^{\prime 2}+v_{z}^{\prime 2}\right) / 3}$, where $v_{i}^{\prime}$ is the standard deviation of a component of the particle velocity within each bin. While this does not represent faithfully the fluid velocity fluctuations, one can observe a transition from a flat low-amplitude profile at low Reynolds number towards a highfluctuation level at high Reynolds number, with a peak in the median plane due to the strong shearing motions. The particle velocity fluctuation magnitude is as expected a strong function of the Reynolds number, at any given location of the vessel. One can note that for the lowest Reynolds number considered, while the flow is expected to be laminar, with $v^{\prime}=0$ everywhere for a flow tracer, the large particle displays nonzero velocity fluctuations. This is a clear signature of the large particles' inertia. 

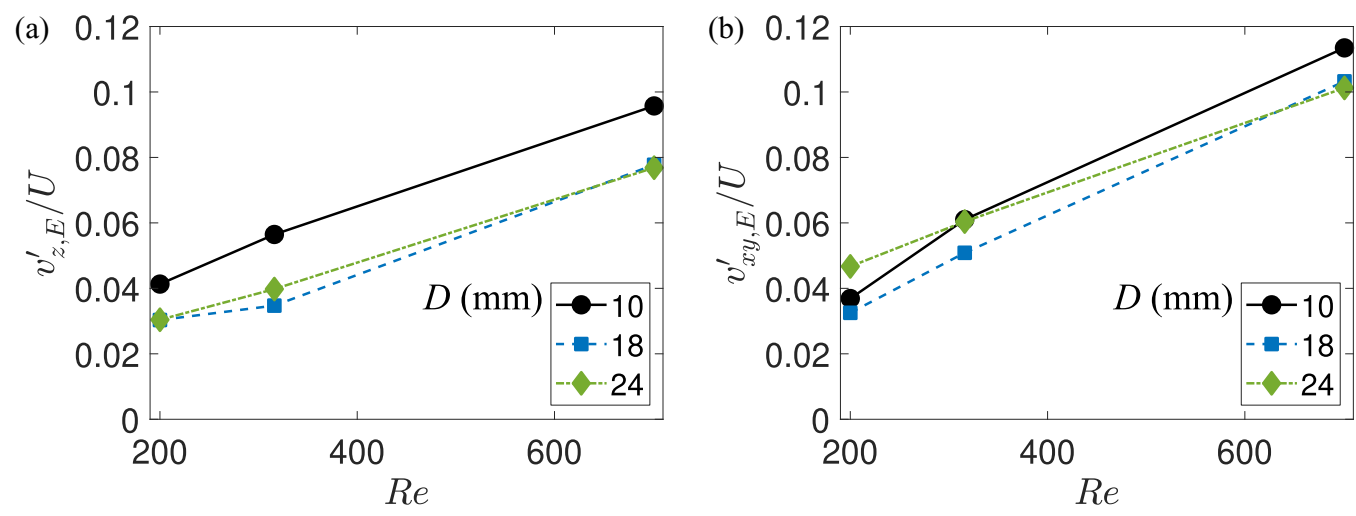

FIG. 5. Standard deviation of the particle velocity fluctuations, after subtraction of a local Eulerian mean along the trajectories, for the (a) axial and (b) transverse components, averaged over both the $x$ and $y$ velocity components, as a function of Re, for each particle diameter. For comparison, the values for fully developed turbulent conditions, found independently of Re and $D$, are $0.13 \mathrm{U}$ and $0.15 \mathrm{U}$ for the axial and transverse components, respectively.

Figure 5 presents an integration across the vessel of the particle velocity fluctuations, giving an overall measure of the particle fluctuation level. This quantity is obtained by defining trajectories of the particle velocity fluctuation, where the local mean particle velocity is subtracted, using the information of the Eulerian conditioning [7,11]. The standard deviation of all the samples from these fluctuation trajectories yields a particle fluctuation that is not biased by the mean flow structure and will be denoted by the index $E$ as the Eulerian local mean particle velocity was subtracted along the trajectories. Figures 5(a) and 5(b) report the axial and transverse components of the unbiased particle velocity fluctuations $v_{z, E}^{\prime}$ and $v_{x y, E}^{\prime}$, respectively (the latter being averaged over both $x$ and $y$ components, both being statistically identical). Both components show a qualitatively linear increase of the fluctuation magnitude with the Reynolds number, roughly doubling when Re increases from 200 to 701, which is purely due to a decrease in the fluid's viscosity as the disk rotation speed $\Omega$ is kept constant. For reference, at $\operatorname{Re}=225000$, the particle velocity fluctuations are equal to $v_{z, E}^{\prime}=0.13 U$ and $v_{x y, E}^{\prime}=0.15 U$.

The particle velocity fluctuations, unlike the mean velocity, depend on particle size, which is consistent with the case of fully developed turbulence [11]. The magnitude of the unbiased particle velocity fluctuations $v_{E}^{\prime}=\sqrt{\left(v_{x, E}^{\prime 2}+v_{y, E}^{\prime 2}+v_{z, E}^{\prime 2}\right) / 3}$ for a 10 -mm particle is about 1.3 times the one of either 18- or 24-mm particles. However, the dependence is stronger on the axial than on the transverse component, in contrast to the case of fully developed turbulence. This indicates that the size dependence of the velocity fluctuations is, for these low-Reynolds-number conditions, a consequence of the particle trapping, which will be discussed in the next section.

\section{PARTICLE TRAPPING}

While some degree of preferential sampling was observed for fully developed turbulent conditions, this phenomenon is largely amplified as the Reynolds number decreases. The probability of finding a large particle on the sides of the vessel strongly increases and the regions that are preferentially sampled by the particles become smaller and smaller in size. This was already observed with the standard deviation of the absolute value of the particle axial position [Fig. 2(d)] and is confirmed by the 2D probability density function (PDF) of the particle position in Fig. 6 . Once again, better statistical convergence is reached by an azimuthal integration of the 3D Cartesian statistics, so $\operatorname{PDF}(r / R, z / R)$ is displayed for an 18-mm particle. For Re $=200$ and 316, the PDFs are not symmetrical, which may be attributed to the fact that the trapping is so strong here that we 

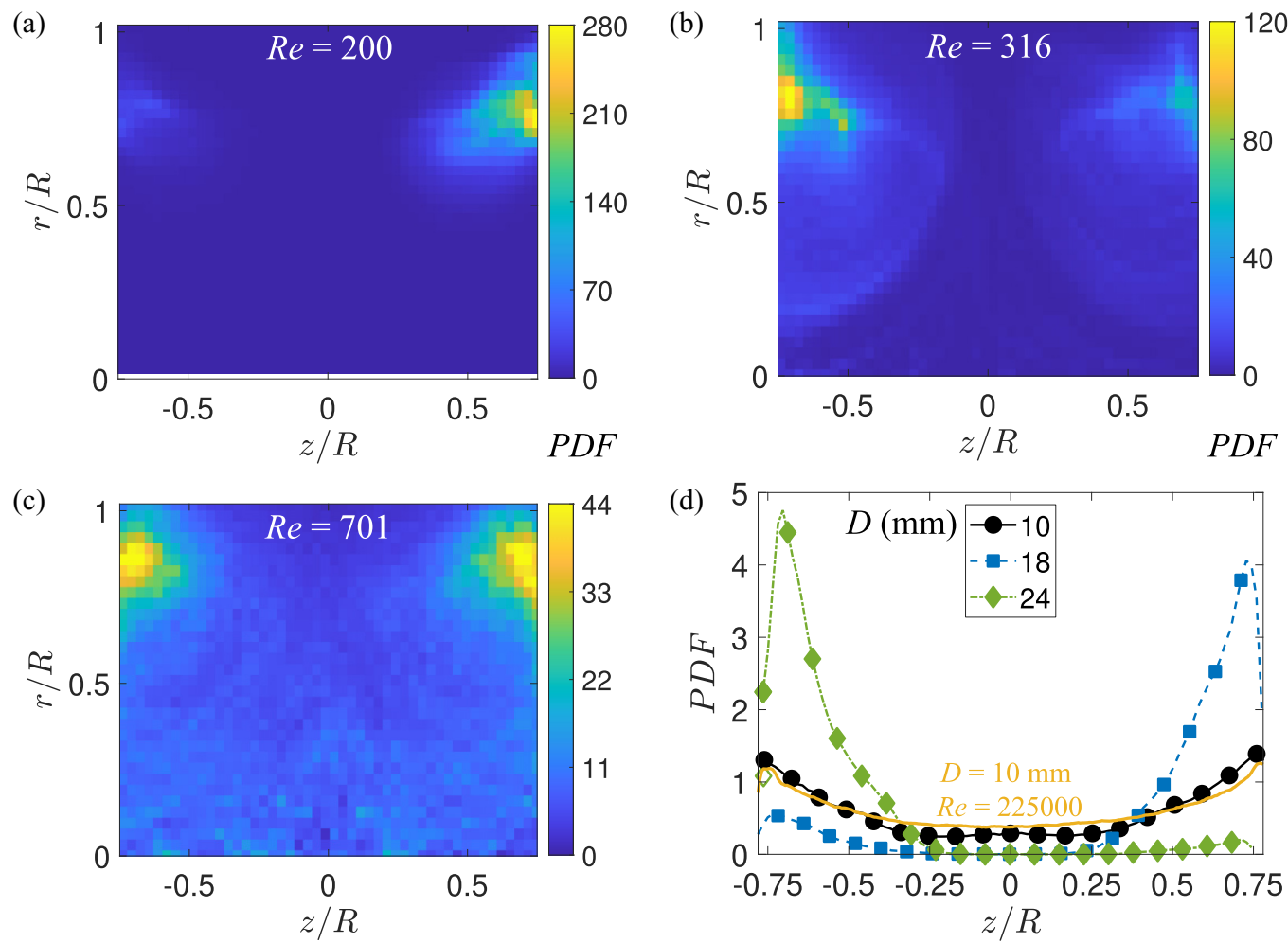

FIG. 6. (a)-(c) Probability density functions of the particle position for a diameter of $18 \mathrm{~mm}$ and the three considered Reynolds number. The PDFs are integrated azimuthally to increase statistical convergence, changing the frame of reference from Cartesian $(x, y, z)$ to cylindrical $(r, \theta, z)$ for this figure. (d) Axial position PDF for $\operatorname{Re}=200$ and the three particle diameters considered (only one-fifth of the symbols are shown for better visibility). The PDF for a 10-mm particle in the fully developed turbulent flow is added for comparison (solid line). The probability to be on the sides of the vessel (corresponding to $|z|>0.25 R$, half of the vessel's volume) goes from $75 \%$ to $61 \%$ for $D=10 \mathrm{~mm}$ when Re increases, while it goes from $92 \%$ to $69 \%$ for $D=24 \mathrm{~mm}$.

do not have enough independent realizations of the particle dynamics to fully converge the PDF in each bin. This is due to the extreme scale difference between the particle dynamics timescales and its typical residence times on a given side of the vessel. For the lowest Reynolds number considered, the 18-mm particle was only observed to stay on the $z<0$ side of the vessel for the whole movie duration (about 30 s) 22 times, while being seen 146 times on the other side, and only two movies show an excursion from a given side to the opposite side of the vessel. A striking difference from the turbulent conditions, where the preferential sampling was mostly happening solely along the axial $z$ coordinate, is that for low-Reynolds-number conditions, preferential sampling also happens along the radial $r$ coordinate. The most explored area across the Reynolds number range is in the vicinity of $(z / R, r / R)=(0.7,0.8)$. These regions correspond to the flow islands, where no fluid's particle can escape from in the case of laminar conditions [the location of the maximum of the axial position PDF is in good agreement with the neutral line locations, seen in the vicinity of $z=0.7 R$ in Fig. 2(f)]. The preferential sampling is such that it will be referred to as particle trapping. In the range of Reynolds numbers explored, the large particles avoid not only the shear layer in the median plane, but also the jet regions created by the disk pumping in the vicinity of the $z$ axis $[(r=0, z)$ in cylindrical coordinates]. The probability to find an 18-mm particle in the region defined as $r>0.7 R$ (representing a third of the vessel total volume) is $80 \%, 60 \%$, and $40 \%$ for $\operatorname{Re}=200,316$, and 
701, respectively. In contrast, the $10-\mathrm{mm}$ particle only samples this region about $40 \%$ of the time, independently of the Reynolds number.

Figure 6(d) shows how particle trapping varies with the particle diameter $D$ for the lowest Reynolds number considered, where it is the strongest, and only the islands are seemingly sampled by the $18-\mathrm{mm}$ particle. The $2 \mathrm{D}$ PDFs are integrated along $r$ to simply compare $\operatorname{PDF}(z / R)$ and study the axial observation of the flow. The PDFs are almost identical for 18- and 24-mm-diam particles, the peak on a given side having a similar shape, with only a slightly larger amplitude for $24 \mathrm{~mm}$ (4.8 versus 4.1). We provide the PDF of a 10-mm particle at $\mathrm{Re}=225000$ as a reference for homogeneous sampling of a closed system. The shape of the PDF, with a probability about only 4 times greater near the edge (but without peaks) than in the center, is due to the slow particle response time. This is a confinement effect which is observed in many closed systems with correlated motion, and only white noise fluctuations would yield a flat PDF [30,31]. Upon arrival in the vicinity of the vessel boundary, it takes time for the particle to change its velocity direction and move away from it. At the lowest Reynolds number explored, the PDF is almost identical for a 10-mm particle to the one for fully developed turbulence. This means that the criterion for particle trapping at low Reynolds number, a function of particle size, is similar if not identical to the size criterion for preferential sampling found for turbulent conditions.

While the sampling of the flow of a 10 -mm particle is quasi-identical for $200 \leqslant \operatorname{Re} \leqslant 701$ and for $\operatorname{Re}=225000$, one may wonder about the timescales associated with the motion of the particle across the vessel. Each trajectory is separated between times spent on a given side of the vessel [discriminating by the sign of $Z(t)$ ], yielding a collection of residence times $\Delta T$. After removal of the residence times obtained from the start and end of each trajectory, which only represent a lower bound to the actual values, we compute the PDFs, which are exponentially decreasing, in the form $\operatorname{PDF}(\Delta T) \propto \exp \left(-\Delta T / T_{0}\right)$, where $T_{0}$ defines a characteristic residence time [Fig. 7(a)]. Although the $10-\mathrm{mm}$ particle sample the flow in a roughly homogeneous manner independently of the Reynolds number, as its velocity fluctuation magnitude only varies with Re (or rather with $v$ ), residence times are a strong function of the Reynolds number. At $\operatorname{Re}=200$, although with a very weak probability, one can observe times up to $20 \mathrm{~s}$ (two-third of the acquisition time; one may note that no movie was ever observed where the $10-\mathrm{mm}$ particle fully stays on a given side). In comparison, the residence times at $\mathrm{Re}=701$ never exceed $7 \mathrm{~s} \mathrm{(70 \%} \mathrm{of} \mathrm{the} \mathrm{acquisition} \mathrm{time).} \mathrm{The}$ characteristic residence time $T_{0}$ decreases approximately threefold as Re increases, in a fashion that may be deemed asymptotic, as $T_{0} \simeq 1 \mathrm{~s}$ for $\mathrm{Re}=225000$.

No residence time can be computed with the current data for particles larger than $10 \mathrm{~mm}$. In an attempt to compute a characteristic timescale of the particle dynamics within the vessel, we turn toward autocorrelation functions of the particle position. They are defined as $R_{i}(\tau)=$ $\langle i(t) i(t+\tau)\rangle /\left\langle i^{2}\right\rangle$, where $i=\left(x^{\prime}, y^{\prime}, z^{\prime}\right)$ are the particle position fluctuations and $\tau$ is a time lag, and they characterize how fast information of previous positions is forgotten in the particle dynamics, mixing exploration of a given side of the vessel and possible existing travels to the opposite side. If a significant amount of back-and-forth motions was observed, the axial autocorrelation function $R_{z}$ would reach 0 at times shorter than the acquisition time, which is not the case here. Assuming that escaping the island can be made by accumulating incremental displacement, slowly displacing the particle out of the stable orbit, we study instead the transverse position autocorrelation function $R_{x}$ [Figs. 7(c) and 7(d)]. Due to the nature of the large particle trajectories, the function oscillates, approximately at the identified frequency $f=0.259 \Omega=1.04 \mathrm{~Hz}$ for the three Reynolds numbers considered (with a slight dependence on the Reynolds number, as the oscillations slowly get out of phase at long times). The oscillations are contained in an exponentially decreasing envelope, which governs the pace at which decorrelation can happen at long time lags. A fit of this exponential decrease yields a characteristic time, denoted by $T_{x}$ and reported in Fig. 7(b). The correlation time $T_{x}$ decreases from about $90 \mathrm{~s}$ to $0.9 \mathrm{~s}$ when Re increases from 200 to 701 , independently for 18- and 24-mm-diam particles. This time is much smaller for the smaller particle, by a factor of 7.5 at $\operatorname{Re}=200$, indicating a richer dynamics, where the particle can escape the orbit much faster and much more often. 

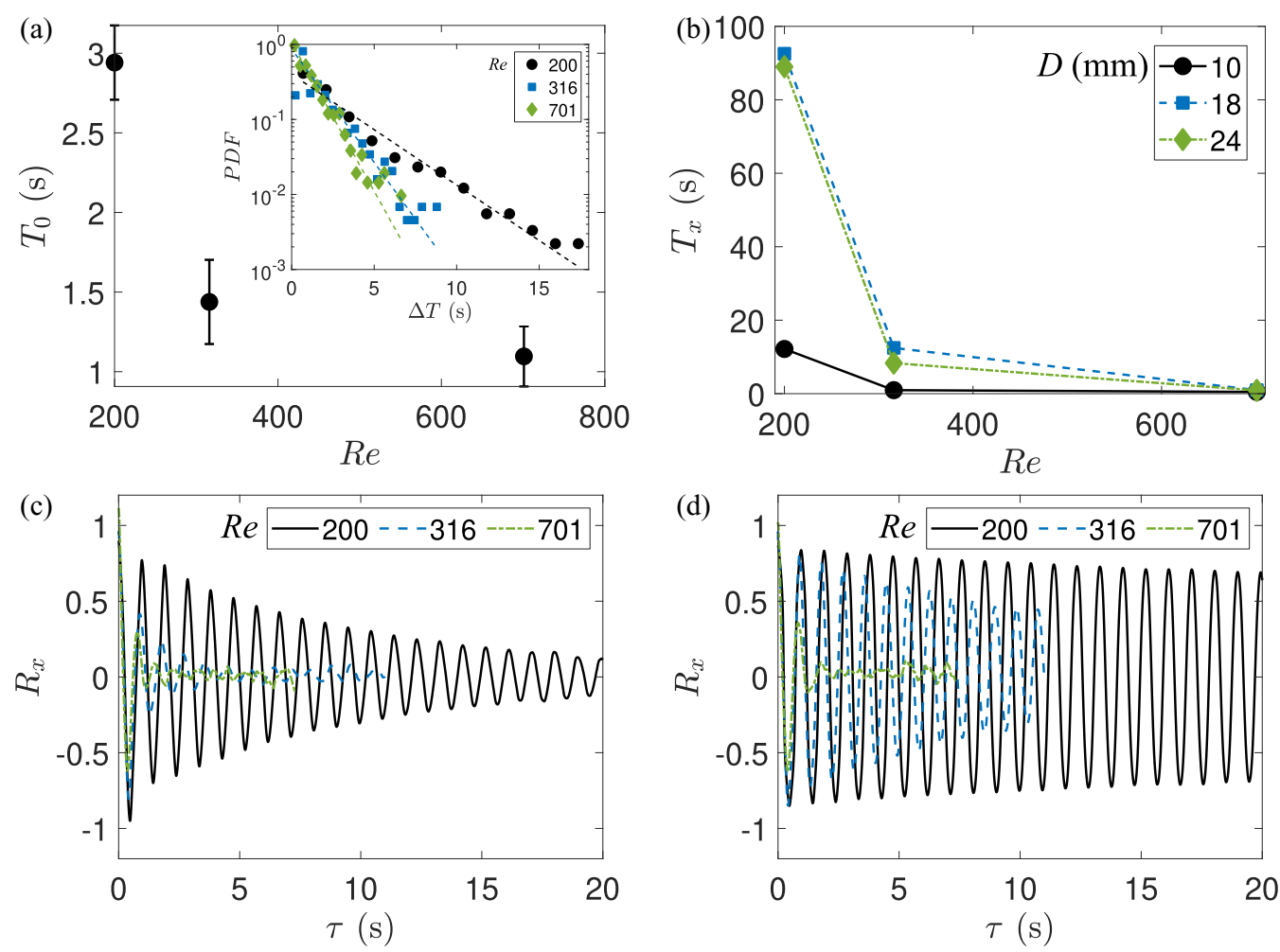

FIG. 7. Characteristic residence time $T_{0}$ on one side of the vessel for a particle of $10 \mathrm{~mm}$ and as a function of the Reynolds number. The time is extracted by fitting the exponentially decreasing PDF of the residence time $\Delta T$ (see the inset). We use the $95 \%$ confidence bound to estimate the error bar for the extracted values of $T_{0}$. (b) Correlation time of the transverse position $T_{x}$ as a function of the Reynolds number. The time is extracted by fitting the exponential decrease of the envelope of the autocorrelation functions of the transverse position $R_{x}$. For $\mathrm{Re}=701, T_{x} \simeq 0.5 \mathrm{~s}$ for $10 \mathrm{~mm}$ and is roughly twice this value for both 18 and $24 \mathrm{~mm}$. Also shown is the autocorrelation function of the transverse position $R_{x}$ as a function of the time lag $\tau$ for a (c) 10- and (d) 18-mm particle for the three values of the Reynolds number considered.

\section{DISCUSSION}

This study reveals that for moderate Reynolds numbers, in a transition regime between fully laminar and turbulent conditions in a closed vessel, large particles experience trapping on long times on islands of the large-scale flow. The $10-\mathrm{mm}$ particle samples the flow almost homogeneously while particles of and beyond $18 \mathrm{~mm}$ experience trapping. It appears that the main criterion for the existence of the trapping is the particle size, whereas the trapping intensity is a strong function of the Reynolds number through change of the fluid's viscosity. This is reminiscent of the preferential sampling of such particles in the same configuration, but for fully developed conditions, where, while lighter particles present longer residence times than heavier ones of the same diameter, the criterion for existence of preferential sampling solely depends on particle size and even happens for neutrally buoyant particles [11]. For this case, arguments based on an attracting force emanating from the mean flow structure and mean velocity gradient, balanced with the particle acceleration fluctuations, a strong function of particle size, can qualitatively explain the origin of the critical size.

In the current case, where negligible particle acceleration fluctuation is observed, the value of the unbiased norm of the particle velocity fluctuations $v_{E}^{\prime}=\sqrt{\left(v_{r}^{\prime 2}+v_{\theta}^{\prime 2}+v_{z}^{\prime 2}\right) / 3}$ for the $10-\mathrm{mm}$ 
particle is only about 1.3 times the one of either 18 - or $24-\mathrm{mm}$ particles. This size dependence is not enough to be compatible with the criterion for the existence of the trapping. This is less of an effect than $v_{E}^{\prime}$ doubling when the Reynolds number increases through change of the fluid's viscosity. We conclude that it cannot explain the trapping and is more likely to be a consequence of it. Indeed, the size dependence of $v_{E}^{\prime}$ mostly comes from its axial component, and $v_{z, E}^{\prime}$ being stronger for $10 \mathrm{~mm}$ than for 18 and $24 \mathrm{~mm}$ is compatible with the fact that this particle samples much more the median plane, which is the highest-velocity fluctuation area [Fig. 4(b)], as well as the jet regions created by the disks' pumping.

While a finer study of particle diameter would be needed, the critical size lies in between 10 and $18 \mathrm{~mm}$. A striking finding of this study is that the dynamics above it are, through all the metrics computed here, qualitatively similar and most notably widely different from the dynamics below it. Indeed, large-scale quantities such as the fluctuation of the particle position and velocity are approximately equal across the range of Reynolds numbers for 18- and 24-mm particles and their dynamics show identical spectra and correlation functions, leading to similar timescales. This is unlike the case of large particles in a fully developed flow where velocity fluctuations continuously decrease with particle size. The particles cannot follow motions at scales comparable to their diameter as their size increases, which are indeed energy-containing scales when $D$ is smaller but of the order of the integral length scale of the flow [11]. For transitional flows, where a turbulent cascade is not present, one can expect energy to exist at the large scale associated with the mean flow structure and in smaller-scale bursts of turbulence, as is typically seen in the canonical Poiseuille or Couette flows [32]. One possible explanation for the particle metrics being similar above the critical size could then be that the majority of the length scales of these bursts, often referred to as spots, lie in between 10 and $18 \mathrm{~mm}$ and/or if the majority of the lifetimes of such bursts are smaller than the response time of the larger particles.

One question that can arise is whether increasing the particle response time, through an increase of its size, affects its Lagrangian chaos. The power spectral densities of the 18-mm particle, presenting many well-defined peaks, which can be qualitatively linked to the particle orbits around the $z$ axis and the frequency doubling observed for the axial component PSD, are most certainly associated with a quasiperiodic behavior. We do not deem them to be signatures of Lagrangian chaos. For the same Reynolds number, the 10-mm-particle PSD, while still harboring the three dominant peaks, presents a much smoother function, similar to the 18-mm PSD at higher Reynolds number. This could be interpreted as the particle experiencing more chaos, leading to a better scale continuum. However, we believe this is rather a consequence of the particle escaping the trapping in the vicinity of the island and being able to explore regions of the flow having stronger fluctuations, such as the median plane and the jet regions along the $z$ axis ( $r$ close to 0$)$. The strong dependence of the residence time on the fluid's viscosity points toward a different exploration of the sides of the vessel by a smaller particle. Instead of mostly orbiting around the $z$ axis with $r=(0.8 \pm 0.1) R$, the particle can freely sample regions with $r<0.8 R$ and fully explore the axial extent of the vessel side. This is confirmed by the standard deviation of the axial position $\left|Z^{\prime}\right| / R$ being almost equal to one-quarter of the vessel axial extent [Fig. 2(d)]. For the lowest Reynolds number, the fluctuation level on a side of the vessel is, most of the time, not sufficient to cross the shear layer of the median plane and the particle is pushed back toward the island. As the Reynolds number increases, so does the particle velocity fluctuation [Fig. 4(b)], so motions through the median plane become more and more frequent, decreasing the characteristic time $T_{0}$, close to its value in fully developed turbulence for $\operatorname{Re}=701$.

While the critical size value that determines trapping cannot be estimated exactly from the present study, some argument can be given concerning its existence. It seems clear that the trapping is associated with the islands of the large-scale flow structure. Let us assume a particle initially located within the island, orbiting around the $z$ axis, to analyze how it could escape it and what trapping mechanism could be involved. As stated above, the particle velocity fluctuations depend much more strongly on the flow viscosity than the particle size, so it cannot be the origin of an escaping trapping condition. The existence for trapping seems indeed to be independent of the fluid's viscosity and to 
depend on particle size only. A metric that depends strongly on particle size, or rather that clearly discriminates particles below or above the critical size, is $T_{x}$. The correlation time of the transverse position can be seen as a proxy for how fast the particle can escape the orbits, from incremental change in its radial distance from the $z$ axis. It can also relate to incremental changes in the azimuthal velocity, as velocity and position autocorrelation are linked to each other and the velocity fluctuation correlation time can indeed be seen as a function of $T_{x}$, as here changes in velocity fluctuations are likely to relate to changes of sampled regions. If the particle is slightly displaced off the orbit, it will have a velocity difference from the local fluid velocity due to its response time. The particle velocity will be greater than the fluid's velocity, as the island is where the azimuthal velocity is the strongest, increasingly as the particle response time increases (through a size increase here). A simplified view is that of a particle in an incoming shear flow, with greater velocity on the edge of the particle further away from the island and smaller velocity on the side closer to the island. Assuming a displacement of the order of $0.1 R$, compatible with the amplitude of the axial motion within a side of the vessel [Fig. 2(d)], and assuming the velocity of the particle is roughly unchanged from the one it had when in the center of the island, this yields a velocity difference of about $\Delta V \simeq 0.02 U$, corresponding to a particle Reynolds number $\operatorname{Re}_{p}=\Delta V D / v>1$. This places the flow around the sphere beyond a creeping flow regime, and the shear magnitude is of the order of $0.2 U / R$. In this regime, the shear-induced lift force is directed toward the lower-velocity region, meaning back toward the island [33-36]. This qualitative explanation would then be in agreement with the finding for the existence of the trapping, in the form of an attractive force toward the flow's islands, which strongly increases with particle size. Smaller particles, through their smaller diameters and response times, experience a much smaller shear-induced lift force and manage to quickly escape this trapping force, while particles above a critical size have their dynamics largely governed by it.

\section{CONCLUSION}

We have presented an experimental study of the dynamics of large particles in a closed swirling flow, in a transitional regime between laminar and turbulent conditions. Three particle diameters and three Reynolds numbers, varied by a change of the fluid's viscosity, keeping the mean flow structure velocity magnitude constant, were studied. The most important finding is the existence of a critical size whose value lies between 10 and $18 \mathrm{~mm}$, with the particle dynamics above it being qualitatively similar, but widely different from below it. A first observation, which can simply be made by looking at the sampling of the vessel from the particles, is that the $10-\mathrm{mm}$ particle explores it in a very homogeneous fashion, which is independent of the fluid's viscosity. In contrast, larger particles, namely, 18 and $24 \mathrm{~mm}$, are found most of the time trapped in a small subset of the flow's volume, which are in the vicinity of flow islands. They correspond to the surroundings of the poloidal neutral lines, closed orbits located around $|z|=0.7 R$ and $r=0.75 R$, where the fluid's particles are trapped indefinitely in fully laminar conditions. While the existence criterion for trapping is solely dependent on the particle size, its magnitude is a strong function of the fluid's viscosity, with the large particles sampling approximately a twice bigger region when the Reynolds number increases from 200 to 701. At the smallest Reynolds number, the dynamics for large particles is quasiperiodic, while at the largest one, fluctuations lead to a smooth spectrum, with the emergence of a long-time dynamics corresponding to back-and-forth motions between each side of the vessel. We note that while particle velocity fluctuations present a roughly linear increase with the fluid's viscosity, the average particle velocity is found to be independent of both size and viscosity and displays similar values as those for fully developed turbulent conditions.

A tentative explanation is made to explain the existence of a trapping criterion and why it solely depends on the particle diameter. The qualitative mechanism proposed finds its origin in the velocity difference between the large particle and the fluid, leading to a shear-induced lift force, a quadratic function of particle size, strong enough for large particles to trap them on the flow's islands. Future work could include a study with finer particle diameter increments, and it would be interesting to compare the dynamics at a different fluid's density, as a dependence of the critical size on 
$\rho_{f}$ would support the lift force scenario. We believe that the results of the present study are general to the behavior of large particles in any transitional flow with the presence of a large-scale structure. Taylor-Couette systems or Rayleigh-Bénard convection immediately come to mind, but it is also the case of natural applications such as transport of waste in underwater currents.

\section{ACKNOWLEDGMENTS}

This research was supported by the French National Research Agency Program No. ANR-16CE30-0028.

[1] J. M. Ottino, Mixing, chaotic advection, and turbulence, Annu. Rev. Fluid Mech. 22, 207 (1990).

[2] F. Toschi and E. Bodenschatz, Lagrangian properties of particles in turbulence, Annu. Rev. Fluid Mech. 41, 375 (2009).

[3] N. M. Qureshi, U. Arrieta, C. Baudet, A. Cartellier, Y. Gagne, and M. Bourgoin, Acceleration statistics of inertial particles in turbulent flow, Eur. Phys. J. B 66, 531 (2008).

[4] S. Klein, M. Gibert, A. Bérut, and E. Bodenschatz, Simultaneous 3D measurement of the translation and rotation of finite-size particles and the flow field in a fully developed turbulent water flow, Meas. Sci. Technol. 24, 024006 (2012).

[5] M. Cisse, H. Homann, and J. Bec, Slipping motion of large neutrally buoyant particles in turbulence, J. Fluid Mech. 735, R1 (2013).

[6] M. Cisse, E.-W. Saw, M. Gibert, E. Bodenschatz, and J. Bec, Turbulence attenuation by large neutrally buoyant particles, Phys. Fluids 27, 061702 (2015).

[7] N. Machicoane and R. Volk, Lagrangian velocity and acceleration correlations of large inertial particles in a closed turbulent flow, Phys. Fluids 28, 035113 (2016).

[8] O. Liot, F. Seychelles, F. Zonta, S. Chibbaro, T. Coudarchet, Y. Gasteuil, J.-F. Pinton, J. Salort, and F. Chillà, Simultaneous temperature and velocity Lagrangian measurements in turbulent thermal convection, J. Fluid Mech. 794, 655 (2016).

[9] V. Mathai, X. Zhu, C. Sun, and D. Lohse, Flutter to tumble transition of buoyant spheres triggered by rotational inertia changes, Nat. Commun. 9, 1792 (2018).

[10] Y. Wang, A. Sierakowski, and A. Prosperetti, Rotational dynamics of a particle in a turbulent stream, Phys. Rev. Fluids 4, 064304 (2019).

[11] N. Machicoane, R. Zimmermann, L. Fiabane, M. Bourgoin, J.-F. Pinton, and R. Volk, Large sphere motion in a turbulent swirling flow, New J. Phys. 16, 013053 (2014).

[12] N. Machicoane, M. López-Caballero, L. Fiabane, J.-F. Pinton, M. Bourgoin, J. Burguete, and R. Volk, Stochastic dynamics of particles trapped in turbulent flows, Phys. Rev. E 93, 023118 (2016).

[13] T. Barois, P. D. Huck, M. Bourgoin, and R. Volk, Equilibrium position of a rigid sphere in a turbulent jet: A problem of elastic reconfiguration, Phys. Rev. E 96, 033105 (2017).

[14] L. A. Spielman, Particle capture from low-speed laminar flows, Annu. Rev. Fluid Mech. 9, 297 (1977).

[15] A. Guha, Transport and deposition of particles in turbulent and laminar flow, Annu. Rev. Fluid Mech. 40, 311 (2008).

[16] P. Matulka, X. Du, and P. Walzel, Particle motion and separation in a laminar tube flow with downstream enlargement, Chem. Eng. Sci. 66, 5930 (2011).

[17] V. Loisel, M. Abbas, O. Masbernat, and E. Climent, The effect of neutrally buoyant finite-size particles on channel flows in the laminar-turbulent transition regime, Phys. Fluids 25, 123304 (2013).

[18] F. Ravelet, Bifurcations globales hydrodynamiques et magnétohydrodynamiques dans un écoulement de von Kármán turbulent, Ph.D. thesis, Université Paris 7, 2005.

[19] F. Ravelet, A. Chiffaudel, and F. Daviaud, Supercritical transition to turbulence in an inertially driven von Kármán closed flow, J. Fluid Mech. 601, 339 (2008).

[20] S. Kreuzahler, D. Schulz, H. Homann, Y. Ponty, and R. Grauer, Numerical study of impeller-driven von Kármán flows via a volume penalization method, New J. Phys. 16, 103001 (2014). 
[21] J. G. Franjione, C.-W. Leong, and J. M. Ottino, Symmetries within chaos: A route to effective mixing, Phys. Fluids A 1, 1772 (1989).

[22] T. C. Lackey and F. Sotiropoulos, Relationship between stirring rate and Reynolds number in the chaotically advected steady flow in a container with exactly counter-rotating lids, Phys. Fluids 18, 053601 (2006).

[23] C. Escauriaza and F. Sotiropoulos, Trapping and sedimentation of inertial particles in three-dimensional flows in a cylindrical container with exactly counter-rotating lids, J. Fluid Mech. 641, 169 (2009).

[24] M. R. Maxey, The motion of small spherical particles in a cellular flow field, Phys. Fluids 30, 1915 (1987).

[25] E. Climent, M. Simonnet, and J. Magnaudet, Preferential accumulation of bubbles in Couette-Taylor flow patterns, Phys. Fluids 19, 083301 (2007).

[26] N. T. Ouellette, H. Xu, M. Bourgoin, and E. Bodenschatz, Small-scale anisotropy in Lagrangian turbulence, New J. Phys. 8, 102 (2006).

[27] R. Monchaux, F. Ravelet, B. Dubrulle, A. Chiffaudel, and F. Daviaud, Properties of Steady States in Turbulent Axisymmetric Flows. Phys. Rev. Lett. 96, 124502 (2006).

[28] N. Machicoane, J. Bonaventure, and R. Volk, Melting dynamics of large ice balls in a turbulent swirling flow, Phys. Fluids 25, 125101 (2013).

[29] P. D. Huck, N. Machicoane, and R. Volk, Production and dissipation of turbulent fluctuations close to a stagnation point, Phys. Rev. Fluids 2, 084601 (2017).

[30] H. A. Kramers, Brownian motion in a field of force and the diffusion model of chemical reactions, Physica 7, 284 (1940).

[31] P. Hänggi, P. Talkner, and M. Borkovec, Reaction-rate theory: Fifty years after Kramers, Rev. Mod. Phys. 62, 251 (1990).

[32] Hydrodynamic Instabilities and the Transition to Turbulence, edited by H. L. Swinney and J. P. Gollub, Topics in Applied Physics Vol. 45 (Springer, Berlin, 1981).

[33] T. R. Auton, The lift force on a spherical body in a rotational flow, J. Fluid Mech. 183, 199 (1987).

[34] D. Legendre and J. Magnaudet, A note on the lift force on a spherical bubble or drop in a low-Reynoldsnumber shear flow, Phys. Fluids 9, 3572 (1997).

[35] D. Legendre and J. Magnaudet, The lift force on a spherical bubble in a viscous linear shear flow, J. Fluid Mech. 368, 81 (1998).

[36] E. Loth, Particles, Drops and Bubbles: Fluid Dynamics and Numerical Methods (Cambridge University Press, London, 2010). 